\title{
Pemphigoid nodularis (nicht bullös)
}

\section{Mechtel \\ V. Prugovecki \\ B. Knopf}

\section{Pemphigoid Nodularis (Non Bullous) - A Case Report}

Zusammenfassung

Das bullöse Pemphigoid kann klinisch völlig untypisch verlaufen. Eine der seltensten Varianten ist das noduläre Pemphigoid. Wir berichten über einen Patienten mit dieser seltenen Erkrankung. In unserem Fall zeigte der Patient prurigoähnliche Hautveränderungen. Die direkte Immunfluoreszenz war mit linearen Ablagerungen von IgG und Komplement entlang der Basalmembranzone positiv. Es wurden zirkulierende IgG-Antikörper gegen die Basalmembranzone nachgewiesen und die Antikörper lagerten sich in der „Salt split“-Technik am Blasendach ab. Im Unterschied zu den meisten Fallberichten entwickelte der Patient keine Blasen.
Abstract

Bullous pemphigoid is known to occur in an atypical manner with pemphigoid nodularis being one of the rarest manifestations. We report a patient suffering from this distinct illness. Our patient presented prurigo-like lesions. Direct immunoflourescence was positive with linear basement membrane zone deposits of IgG and complement. Circulating IgG anti-BMZ antibodies were detected and antibodies could be seen at the roof of the bullae in salt split immunofluorescence. In contrast to most reported cases our patient developed no blisters at all.

\section{Kasuistik}

\section{Anamnese}

G.K.: 79 Jahre, männlich, Rentner

Vor 8 Monaten traten erstmals stark juckende, anfangs ekzemartige später knotige Hautveränderungen mit bevorzugter Lokalisation an den Extremitäten auf. Die Effloreszenzen wurden blutig gekratzt und heilten mit Hyperpigmentierungen ab. Ambulant wurde bei fortschreitenden Befunden bis zum Zeitpunkt der stationären Aufnahme mit kortisonhaltigen Externa ohne sichtbare Besserungstendenz behandelt.
Erwähnenswert sind darüber hinaus ein mit oralen Antidiabetika geführter Diabetes mellitus Typ 2 ohne Komplikationen, eine kompensierte KHK, eine Penicillinallergie sowie ein Zustand nach Parathyroidektomie.

\section{Hautbefund bei Aufnahme}

Knotige, hyperpigmentierte, zum Teil glänzende Hautveränderungen in flächiger Ausdehnung an den Streckseiten beider Oberarme, Unterschenkel und am Gesäß. Daneben deutliche Kratzeffloreszenzen und flache Erosionen mit Köbner-Phänomen-ähnlichem Aspekt. Schon abgeheilte Areale stellten sich kaum infiltriert, aber deutlich hyperpigmentiert dar (Abb. 1 u. 2). Blasen wa-

Institutsangaben

Klinik für Hautkrankheiten und Allergologie, Städtisches Klinikum Zwickau

Widmung

Herrn Prof. Knopf zum 60. Geburtstag.

Korrespondenzadresse

Dr. med. D. Mechtel · FA für Haut- und Geschlechtskrankheiten, Städtisches Klinikum Heinrich Braun Zwickau · Karl-Keil-Straße 35 · Postfach 200943 • 08009 Zwickau 


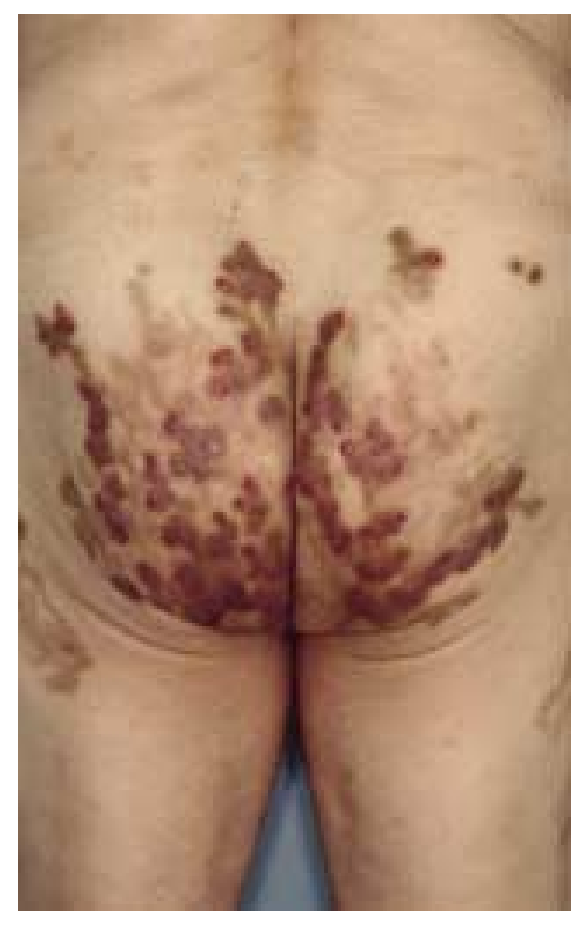

Abb. 1 Detail Gesäß.

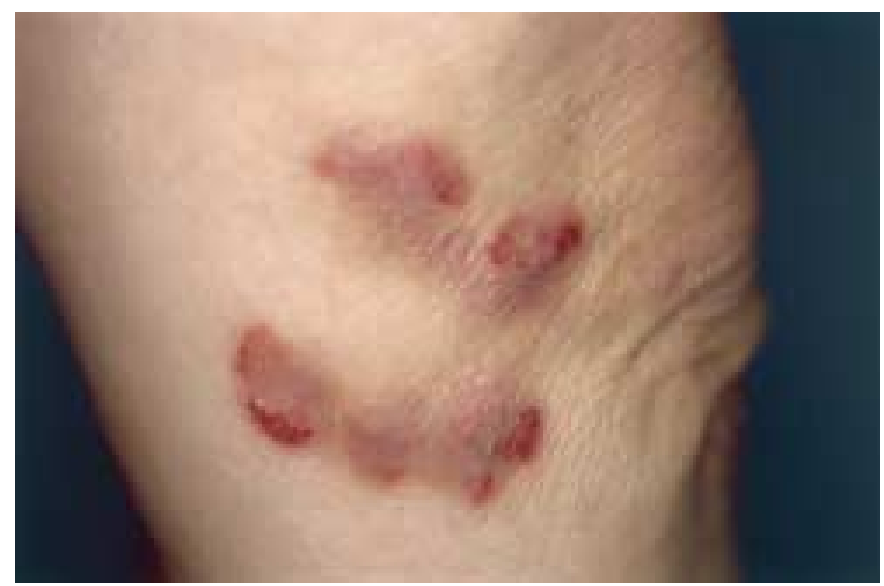

Abb. 2 Detail rechter Oberarm.

ren nicht sichtbar. Alle Schleimhäute waren frei von pathologischen Veränderungen.

\section{Dermatohistopathologie (H 7065/2 vom 3.9.2002, Pathologische Gemeinschaftspraxis, Dr. Neumerkel/ Dr. Otremba, Zwickau):}

Hautweichteilgewebe mit einer akanthotisch verbreiterten Epidermis, die eine oberflächliche lamelläre Parakeratose aufweist, wobei an vielen Stellen eine entzündlich leukozytär-parakeratotische und fibrinös-hämorrhagische Squama vorliegt. Die Reteleisten plump klonal und fingerförmig verlängert und in den basalen Epithelschichten Lymphozyten einschließend. Das Stratum papillare mit Kapillarektasien und unterschiedlich dichten entzündlichen Infiltraten von Lymphozyten mit einzelnen histiozytischen Zellen und beigemischten Granulozyten.

Beurteilung: Hautweichteilgewebe mit chronisch rezidivierter Dermatitis und entzündlicher Squama, wobei die Reaktionsweise teilweise psoriasiform erscheint. Keine Malignität (Abb. 3).

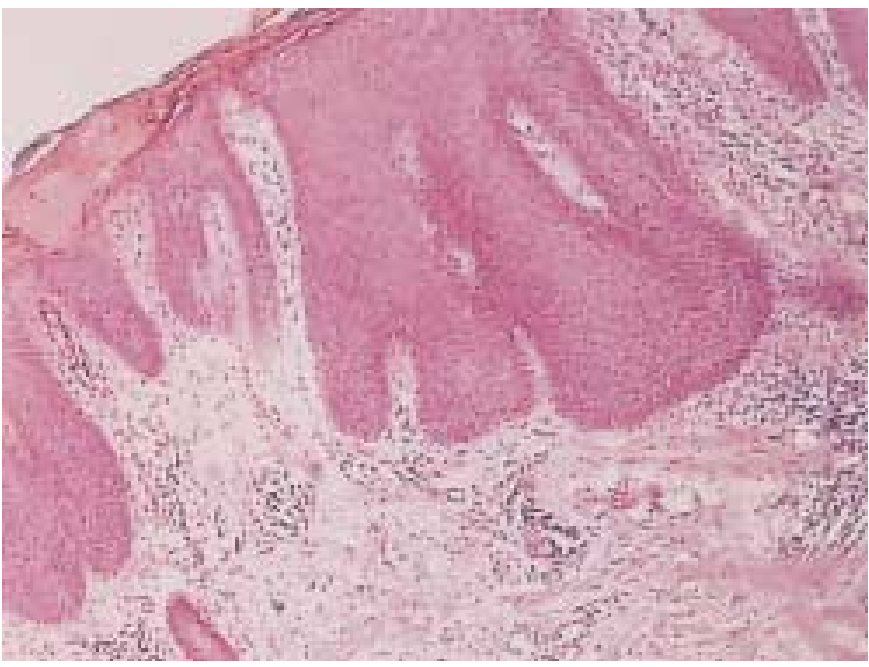

Abb. 3 Histologie: Akanthose, Hyperkeratose und gemischte entzündliche Infiltrate (HE-Färbung, Originalvergrößerung 15fach).

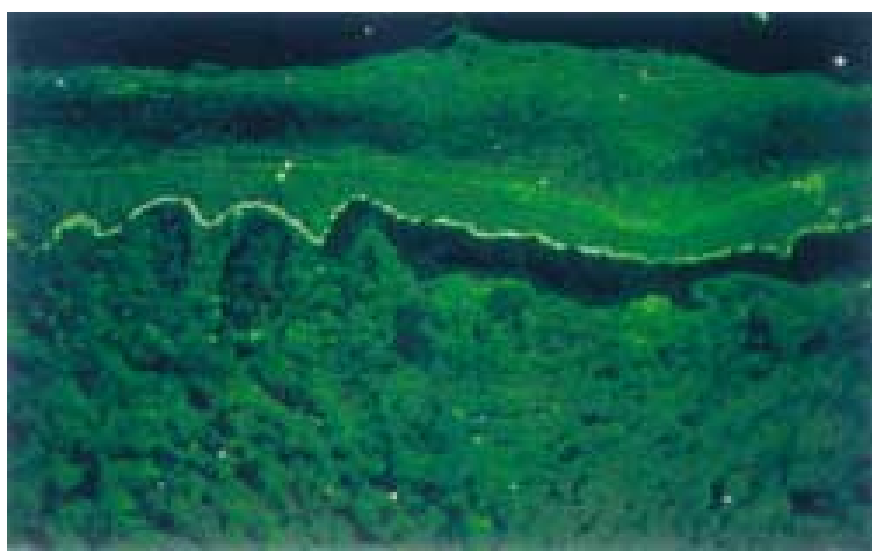

Abb.4 Immunfluoreszenz: Antikörperablagerungen am Blasendach (epidermale Seite des Spaltes).

\section{Laborbefunde}

Direkte IF (Prof. Dr. med. B. Knopf, Klinik für Hautkrankheiten und Allergologie Zwickau): Bei der direkten IF-Untersuchung konnten lineare Ablagerungen von IgG und Komplement entlang der BMZ nachgewiesen werden. Beurteilung: Befund spricht für bullöses Pemphigoid.

AK-mapping - „Salt split“-Technik: Antikörperablagerungen am Blasendach (epidermale Seite des Spaltes) sichtbar (Abb.4).

Beurteilung: bullöses Pemphigoid

Indirekte IF (Prof. Dr. med. B. Knopf, Klinik für Hautkrankheiten und Allergologie Zwickau):

Epidermale Basalmembran-Antikörper (Titer): 1:1280 positiv Epidermale Interzellularsubstanz-Antikörper (Titer): negativ ANF-Titer: negativ ds-DNS-Titer: negativ

Endomysium-Antikörper (Titer): negativ 
Beurteilung: Befund spricht für bullöses Pemphigoid.

ELISA mit rekombinantem BP180 NC16A (Klinik und Poliklinik für Hautkrankheiten der Universität Würzburg, Autoimmunologisches Labor, Prof. Dr. med. Eva Bröcker): 74,2 U/ml (Norm: unter 9 ) positiv

\section{Weitere Laborbefunde:}

BSG: $5 / 20 \mathrm{~mm} / \mathrm{h}$

Blutbild und Differentialblutbild: Monozyten 14\%, sonst unauffällig.

Analyse: $\gamma \mathrm{GT} 1,52 \mu \mathrm{mol} / \mathrm{ls}$, keine weiteren pathologischen Befunde einschließlich Immunelektrophorese

Zirkulierende Immunkomplexe: 108,0 $\mu \mathrm{g} / \mathrm{ml}$ (Norm: unter 55,0)

p-/c-ANCA: negativ

HbA1c: 6,4\% (Norm: 4,4-6,4)

Urinstatus und -sediment: unauffällig

Mykologische Untersuchungen:

- Abstrich Unterschenkel: Präparat negativ, Kultur Candida albicans nach Anreicherung.

- Stuhl I-III: Candida albicans, Keimzahl 1 Mpt/l

Bakteriologische Untersuchungen Abstrich Unterschenkel: Staph. aureus, Resistenzen bei Penicillin und Ampicillin

Stuhluntersuchungen auf Helminthen und Wurmeier: negativ

\section{Gerätetechnische Untersuchungen}

Abdomensonographie: Steatosis hepatis, keine Organinfiltrate. Cholezystolithiasis, Nierenzyste links. Keine paraaortalen Infiltrate.

Röntgen des Thorax: keine frischen Infiltrate.

\section{Therapie und Verlauf}

Wegen des bestehenden Diabetes mellitus wurde zunächst eine intensive externe Therapie der Hautveränderungen mit täglicher Applikation von Momethasonfuorat im Wechsel mit einer Basissalbe durchgeführt.

Aufgrund der Progredienz und des schlechten Ansprechens der externen Behandlung während der ersten Tage des stationären Aufenthaltes entschieden wir uns zu einer systemischen Kortisontherapie. Wir begannen mit $1 \mathrm{mg}$ Solu-Decortin H/kg Körpergewicht i.v., das relativ rasch reduziert und am 13. Tag auf die orale Medikation von $30 \mathrm{mg}$ Tagesdosis reduziert wurde. Eine Dysregulation des Diabetes infolge des applizierten Kortikoids trat nicht auf.

Parallel zur systemischen Therapie führten wir bei anamnestisch guter Sonnenlichtverträglichkeit die Bade-PUVA-Therapie mit einer kumulativen Dosis von 6,2 J/ $\mathrm{cm}^{2}$ UVA in 13 Einzelsitzungen durch.
Diese Therapieoption sprach gut an, neue Hautveränderungen traten nicht mehr auf und die noch bestehenden heilten mit Hyperpigmentierungen $a b$.

Die Entlassung in die ambulante Weiterbehandlung erfolgte mit einer Prednisolondosis von $30 \mathrm{mg} / \mathrm{Tag}$. Empfohlen wurde die Reduktion auf $20 \mathrm{mg}$, danach schrittweise alle fünf Tage um $5 \mathrm{mg}$, um dann einen Auslassversuch $\mathrm{zu}$ unternehmen. Ein Reboundphänomen trat nach telefonischer Auskunft des behandelnden Hautarztes bisher nicht auf.

\section{Diskussion}

Provost et al. beschrieben erstmals 1979 ein BP als noduläre Variante bei zwei weiblichen Patienten unter dem klinischen Bild einer Prurigo nodularis [7]. Der Begriff des Pemphigoid nodularis selbst wurde 1981 von Yung et. al. geprägt [10].

Sehr wahrscheinlich handelt es sich bei dem Krankheitsbild um eine eigene Entität, wenn auch in den vorangegangenen Jahren von der Koinzidenz zweier Erkrankungen ausgegangen wurde. In der Pathogenese werden zwei verschiedene Mechanismen diskutiert [6], wobei eine eindeutige Klärung der Rolle der Antikörperbildungen bisher noch nicht vorliegt: 1. Gleichzeitiges Vorkommen von zwei unterschiedlichen Entitäten, nämlich eine Prurigo nodularis und ein bullöses Pemphigoid. 2. Trauma und lokale Entzündung führten zur Verletzung der Basalmembranzone und induzierten die Bildung von Pemphigoid-Antikörpern bei prädisponierten Personen.

Die noduläre nicht bullöse Variante des BP ist charakterisiert durch multiple, juckende exkoriierte Papeln mit bevorzugter Lokalisation an den Streckseiten der unteren Extremitäten. Sie kann sowohl klinische Zeichen der Prurigo nodularis als auch des BP zeigen.

Bei den meisten Patienten wurde initial über das parallele Auftreten von hyperkeratotischen Veränderungen und straffen Blasen berichtet.

In unserem Fall konnten weder anamnestisch noch im klinischen Bild Blasen eruiert werden. Auch histologisch waren weder eine Blasenbildung noch die sonst typischen eosinophilen Infiltrate nachweisbar. Vorliegende Akanthose, Hyperkeratose und gemischte entzündliche Infiltrate sprachen eher für eine Prurigo.

Diskutiert werden könnte, dass die Blasenbildung unbemerkt vom Patienten verlief und schon zur stationären Aufnahme das knotige Vollbild der Erkrankung vorlag. Andererseits ist es denkbar, dass es ohne Therapie nach längerem Verlauf doch noch zur Blasenbildung gekommen wäre.

Sowohl die direkte als auch die indirekte Immunfluoreszenztechniken einschließlich der „Salt split“-Technik waren bei unserem Patienten im Sinne eines bullösen Pemphigoids positiv.

In den meisten Berichten zur nodulären Form des BP wurden zirkulierende IgG-Antikörper gegen die Basalmembranzone gefunden. Über Ablagerungen von IgA-Antikörper an der Basalmembran wurde seltener berichtet $[1,9]$. 
Als Ziel-Antigene konnten im Western-Immunoblot BP230 (intrazelluläre 230-kDa Komponente der Hemidesmosomen) und BP180 (transzelluläres 180-kDa Kollagen, Kollagen XVII) nachgewiesen werden $[1,5,8]$. Zudem gibt es Veröffentlichungen zu zirkulierenden Antikörpern, welche mit der intrazellulären Domäne von Dsg.1, dem Zielantigen beim Pemphigus foliaceus, reagieren [4].

Bei dem vorgestellten Patienten konnten wir als Target der zirkulierenden Antikörper PB180 demonstrieren. Eine Blasenbildung entwickelte sich dennoch nicht.

Der passive Transfer von anti-BP180-Antikörper im Tiermodell ging auch nicht in allen Fällen mit einer Blasenbildung einher [11].

Die Rolle der Antikörper bei der Entwicklung des Juckreizes und des nodulären Verlaufes ist nicht klar.

In unserem Fall würden wir hinsichtlich der Pathogenese die zweite Hypothese, d. h. Induktion durch Pemphigoid-AK-Bildung durch Trauma (Kratzen) favorisieren.

Auffällig war auch ein erhöhter IgE-Titer, wie er an anderer Stelle schon beschrieben wurde [3]. Welche Rolle dieser erhöhte Titer in der Pathogenese spielt, bleibt Spekulation.

Die Behandlung des NP kann sich schwierig gestalten und die Veränderungen sprechen in vielen Fällen auf die alleinige externe Monotherapie mit Steroiden nicht an. Fast alle in der Literatur veröffentlichten Fälle bedurften einer systemischen Therapie mit Kortison als Monotherapie bzw. in Kombination mit Immunsuppressiva oder Dapson [2]. Es ist infolge nicht verwunderlich, dass auch wir eine systemische Kortisontherapie nicht umgehen konnten. Die zusätzlich durchgeführte Bade-PUVA-Therapie wurde vom Patienten prurituslindernd empfunden. Ob dadurch auch die Heilung beschleunigt wurde, ist fraglich.
Wir präsentieren diesen Fall, um darzustellen, dass bei persistierenden pruriginösen knotigen Veränderungen auch eine blasenbildende Dermatose in die differenzialdiagnostischen Überlegungen mit einbezogen werden sollte.

\section{Literatur}

${ }^{1}$ Ameen M, Harman KE, Black MM. Pemphigoid nodularis associated with nifedipine. Brit J Dermatol 2000; 142: 575-576

${ }^{2}$ Cliff S, Holden CA. Pemphigoid nodularis: a report of three cases and review of the literature. Brit J Dermatol 1997; 136: 398 - 401

${ }^{3}$ Gallo R, Parodi A, Rebora A. Pemphigoid nodularis. Brit J Dermatol 1993; 129: 744-745

${ }^{4}$ Fujisawa H, Ishii Y, Tateishi T, Kawachi Y, Otsuka F, Amagai M, Komai A Hashimoto T. Pemphigoid nodularis with IgA autoantibodies against the intracellular domain of desmoglein 1. Brit J Dermatol 2000; 142: $143-147$

${ }^{5}$ Kawahara Y, Matsumura K, Hashimoto T, Nishikawa T. Immunoblot Analysis of Autoantigens in Localized Pemphigoid and Pemphigoid Nodularis. Acta Derm Venereol 1997; 77: 187-190

${ }^{6}$ Powell AM, Albert S, Gratian MJ, Bittencourt R, Bhogal BS, Black MM. Pemphigoid nodularis (non-bullos): a clinicopathological study of five cases. Brit J Dermatol 2002; 147: 343 - 349

${ }^{7}$ Provost TT, Maizc JC, Ahmed AR, Strauss JS, Dobson RL. Unusual subepidermal bullous diseases with immunologic features of bullous pemphigoid. Arch of Dermatol 1979; 115: 156-160

${ }^{8}$ Schachter M, Brieva JC, Jones JCR, Zillikens D, Skrobek Ch, Chan LS, Pemphigoid nodularis associated with autoantibodies to the NC16A domain of BP180 and a hyperproliferative integrin profile. J Am Acad Dermatol 2001; 45: 747-754

${ }^{9}$ Schmidt E, Sitaru C, Schubert B, Wesselmann U, Kromminga A, Bröcker EB, Zillikens D. Subacute prurigo variant of bullous pemphigoid: Autoantibodies show the same specificity compared with classic bullous pemphigoid. J Am Acad Dermatol 2002; 47: 133 -136

10 Yung CW, Soltani K, Lorincz A. Pemphigoid nodularis. J Am Acad Dermatol 1981; 5: 54-60

${ }_{11}$ Zillikens D, Schmidt E, Reimer S. Antibodies to desmogleins 1 and 3 , but not to BP180 induce blisters in human skin grafted outo SCID mice. J Pathol 2001; 193: 117-124

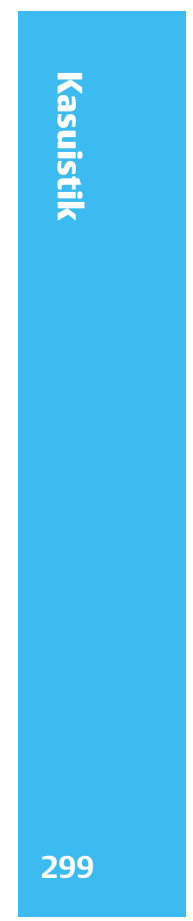

